\title{
Signal amplification of FISH for automated detection using image cytometry
}

\author{
Khuong Truong ${ }^{a}$, Jack Boenders ${ }^{b}$, Zofia Maciorowski ${ }^{c}$, Philippe Vielh ${ }^{c}$, \\ Bernard Dutrillaux $^{a}$, Bernard Malfoy ${ }^{a}$ and Claire A. Bourgeois ${ }^{a, *}$ \\ ${ }^{a}$ Institut Curie - CNRS UMR 147, Paris, France \\ ${ }^{b}$ Becton Dickinson Cellular Imaging Systems BV, Leiden, The Netherlands \\ ${ }^{c}$ Institut Curie - Department of Cytopathology, Paris, France
}

Received 28 October 1996

Revised 27 December 1996

\begin{abstract}
The purpose of this study was to improve the detection of FISH signals, in order that spot counting by a fully automated image cytometer be comparable to that obtained visually under the microscope. Two systems of spot scoring, visual and automated counting, were investigated in parallel on stimulated human lymphocytes with FISH using a biotinylated centromeric probe for chromosome 3. Signal characteristics were first analyzed on images recorded with a coupled charge device (CCD) camera. Number of spots per nucleus were scored visually on these recorded images versus automatically with a DISCOVERY image analyzer. Several fluochromes, amplification systems and pretreatments were tested. Our results for both visual and automated scoring show that the tyramide amplification system (TSA) gives the best amplification of signal if pepsin treatment is applied prior to FISH. Accuracy of the automated scoring, however, remained low (58\% of nuclei containing two spots) compared to the visual scoring because of the high intranuclear variation between FISH spots.
\end{abstract}

Keywords: FISH, interphase cytogenetics, image analysis, spot counting, signal amplification

\section{Introduction}

Fluorescence in situ hybridization (FISH) allows detection of specific DNA and RNA sequences in individual cells [10]. It has become a powerful and sensitive method for chromosomal research, cell biology and especially cytogenetic analysis for diagnostic and prognostic purposes [8,9]. Most of these applications deal with qualitative detection. In the past years, however, with the development of image cytometry, a new tool to evaluate hybridization signals has appeared, allowing quantification of chromosome gains and losses.

The detection and enumeration of FISH spots in interphase nuclei may be done manually using a microscope or automatically using an image cytometer. The image cytometer grabs the image using a CCD camera and then applies a program to detect spots. The manual method is limited in the number of cells counted which leads to errors in detection of small populations of aberrant cells [5].

\footnotetext{
${ }^{*}$ Corresponding author, address: Claire Bourgeois, Institut Curie, UMR 147 CNRS, 26, rue d'Ulm, 75248 Paris Cedex 05, France. Tel.: +33 0142346632; Fax: +33 0142346674; E-mail: cabourg@ curie.fr.
} 
An automated image cytometer allows the analysis of a larger number of nuclei, a procedure which has already been utilized for absorption measurements [3,11].

Some major problems with automated image systems are signal quality, hybridization efficiency and sensitivity of the camera which records the images. We aimed at improvement of the signal, spot size and signal-to-noise ratio, which would allow a more accurate detection of spots. High background noise and small spots often hinder correct segmentation of the specific hybridization signal and therefore lead to incorrect counting of the number of spots per nucleus.

New detection systems which stabilize and amplify the hybridization signal have been developed. New fluochromes which are more photostable and less sensitive to $\mathrm{pH}$ changes in the physiological range compared to the commonly used fluoresceine isothiocyanate (FITC), are now also available. $R$ hodol Green is one of these fluochromes operating in the same excitation and emission range as FITC [13]. In addition, a new amplification system based on the deposition of biotinylated tyramide at the probe location, has been developed [1,2]. Results obtained with this method show a significant amplification of the FISH signal with good localization after special treatments [6,12].

We report here experiments on the effect of pepsin pretreatment combined with the use of different amplification systems. The signal analysis was performed by recording the image with a cooled CCD camera, the subsequent quantification step was performed by a Samba ${ }^{\mathrm{TM}}$ system (Alcatel TITN Answare, Grenoble, France). The same slide preparations were screened on the DISCOVERY ${ }^{\mathrm{TM}}$ system (Becton Dickinson Image Cytometry Systems, Leiden, The Netherlands) in order to evaluate the spot scoring established by the fully automated image cytometer. The tandemly repeated alphoid DNA of the centromeric region of the human chromosome 3 was the target for the FISH on human lymphocytes. Although satellite probes give bright signals, amplification seems to be necessary for automated spot counting. Thus, the present study was done with two fluochromes (FITC and Rhodol Green) and two amplification systems (amplified Rhodol Green and the Tyramide Signal Amplification (TSA)).

\section{Materials and methods}

\subsection{Cell samples}

Short-term phytohemaglutinin-stimulated human lymphocyte cultures were developed according to [4]. Metaphase chromosomes were obtained as followed: briefly, samples treated with colcemid for two hours and hypotonic shock in human plasma diluted in $\mathrm{KCl} 1: 1(\mathrm{v} / \mathrm{v})$, were finally fixed in $3: 1$ methanol: acetic acid (v/v). The preparations were spread over wet-iced cold slides and stored at $-20^{\circ} \mathrm{C}$.

\subsection{Sample pretreatment}

All slides were pretreated with RNase at $37^{\circ} \mathrm{C}$ for one hr, then rinsed twice in $2 \times \mathrm{SSC}$ and once in PBS. The pretreated slides were incubated for $10 \mathrm{~min}$ at $37^{\circ} \mathrm{C}$ in pepsin (Sigma) at $0.5 \mu \mathrm{g} / \mathrm{ml}$ in $0.01 \mathrm{M} \mathrm{HCl}$. They were then washed twice in PBS for $5 \mathrm{~min}$ at room temperature, postfixed in $3: 1$ methanol : acetic acid and air-dried. 


\subsection{In situ hybridization}

The alpha satellite centomeric probe for human chromosome 3, D3Z1 (Oncor-Appligene, Gaithersburg, MD), was applied for FISH.

The centromeric probe was hybridized according to the supplier's instructions: $3 \mu \mathrm{l}$ of probe and $17 \mu \mathrm{l}$ of hybridization buffer were prewarmed at $37^{\circ} \mathrm{C}$ for $5 \mathrm{~min}$, then denaturated at $70^{\circ} \mathrm{C}$ for $10 \mathrm{~min}$. Target DNA was denatured for $3 \mathrm{~min}$ at $70^{\circ} \mathrm{C}$ in $70 \%$ formamide (Fluka), $2 \times \mathrm{SSC}, \mathrm{pH} 7$. Next, slides were rinsed in $2 \times \mathrm{SSC}$, dehydrated in ascending ethanol concentrations and air-dried. The probe mixture was applied and hybridization was performed overnight at $37^{\circ} \mathrm{C}$. Rapid post-hybridization washes were carried out in $0.5 \times \mathrm{SSC}$ at $72^{\circ} \mathrm{C}$ for $5 \mathrm{~min}$.

\subsection{Immunofluorescent detection and signal amplification}

1) For the comparison of FITC and Rhodol Green detection, immunofluorescent detection of the biotin-labeled probe was performed using mouse anti-biotin antibodies (DAKO) followed by FITC or Rhodol Green conjugated anti-mouse IgG antibodies (Molecular Probes).

2) For Rhodol Green amplification, the probe was detected by mouse anti-biotin antibodies (DAKO) followed by a biotinylated antimouse IgG (DAKO). Streptavidin-labeled Rhodol Green (Molecular Probes) was then applied.

3) For the tyramide signal amplification (TSA), the Renaissance TSA Kit (New England Nuclear, MA, USA) was used. After having applied the same order of antibody layers as above (the mouse antibiotin and biotinylated anti-mouse), a streptavidin-labeled antibody coupled to horseradish-peroxydase (HRP) was applied for $30 \mathrm{~min}$ before tyramide deposition. In most experiments, the biotinylated tyramide was used at a concentration of $1 / 250$ of the kit stock solution, different concentrations $(1 / 100,1 / 150,1 / 200$ and 1/250) were then applied to other preparations. Incubation time was always $10 \mathrm{~min}$. Fluorescence detection was achieved by streptavidin-labeled Rhodol Green. Slides were counterstained in 4,6-diamino-2-phenylindole (DAPI, $1 \mu \mathrm{g} / \mathrm{ml}$, Molecular Probes) and mounted in p-phenylene-diamine.

The probe's specifity for the centromere of the chromosome 3 and the hybridization efficiency was verified by manually counting the FISH spots on metaphase spreads which showed specific signals in these regions.

\subsection{Fluorescence signal acquisition and analysis procedure}

1) Visual observations were carried out with an epifluorescence microscope (Axiophot, Zeiss) equipped with a mercury arc lamp and a $100 \times$ oil immersion objective (0.7-1.3 N.A.).

2) Images of metaphases and nuclei were recorded using a cooled dual-mode CCD camera (C4880, Hamamatsu). For each series of slides, integration time was adjusted according to the strongest and weakest signal (between 1.2 and $1.4 \mathrm{~s}$ for FITC excitation) and kept at the same value during the acquisition in order to use the entire grey level range. As it was not possible to work with the same acquisition time from one set of experiments to another, FITC was chosen as a reference for each set to make comparison possible between different experiments. For controls, 10 metaphases were recorded for each sample and for signal quantification, 30-40 nuclei were acquired. These metaphases and nuclei were selected at low magnification (using a DAPI filter at $330 \mathrm{~nm}$ ) on the basis of a well spread morphology. After background subtraction, the images were analyzed with SAMBA $^{\text {TM }} 2640$ software (Alcatel-TITN): FISH spots were segmented, their integrated intensity 
measured and profile shape established, calculating all values on 4096 grey levels. Spot counting was then performed on these images by at least two different observers. Spots were chosen on the basis of: a) size, b) intensity, c) compactness and d) localization within the nucleus. Big bright spots inside the DAPI mask were accepted, whereas small diffuse spots were rejected.

Background values were determined from empty field images which were also used for background subtraction.

\subsection{Automated image analysis}

The spot scoring of the previously analyzed slides was performed using DISCOVERY ${ }^{\mathrm{TM}}$ automated image analyzer (Becton Dickinson Image Cytometry Systems, Leiden, The Netherlands) consisting of a Leitz Autoplan microscope with a $40 \times$ oil objective (numerical aperture: 1,3), a Xillix 1400 black and white camera with a variable integration time from $30 \mathrm{msec}$ to $10 \mathrm{sec}$ and a Matrox Image Series frame grabber connected to a PC Intel 486 computer. The light source used was a mercury arc lamp $(100 \mathrm{~W})$.

The primary objects were segmented in a test field by exciting the DAPI counterstained nuclei at $330 \mathrm{~nm}$. Then, the FISH signals (excited at $485 \mathrm{~nm}$ ) were defined as secondary objects. In order to better focus and detect the spots, the 2D-projection mode was used. This function allows grabbed images at different focal planes with reconstruction of an optimally focused image, losing fewer out-of-plane spots during segmentation. The secondary objects were then automatically matched to the extent of the DAPI mask defining the primary object. Data was acquired in list mode and stored with the corresponding images. 650 nuclei were counted, artifacts or incorrectly segmented objects (representing up to $10 \%$ of the objects) were eliminated interactively by using the gallery display and the resulting histograms were generated by the DISCOVERY ${ }^{\mathrm{TM}}$ software. No interactive reclassification was performed. The assigned gates were 0 spots, 1 spot, 2 spots and more than 2 spots per nucleus.

\section{Results}

\subsection{Visual observations and analysis of recorded images}

The first set of results concerns the efficiency of the hybridization using the centromeric probe. Signals were detected only at the chromosome 3 centromeres and $100 \%$ of the visually observed and recorded metaphases showed two detectable spots (Fig. 1). Manual score of interphase nuclei on the images recorded with the CCD camera (Table 1) showed about $82-100 \%$ of nuclei with two spots. The shape and intensity of the signals, however, was very heterogeneous within a nucleus and between different nuclei. This visual observation is confirmed by data shown in Fig. 2. The general distribution of integrated fluorescence (Fig. 2a) and area of the spots (Fig. 2b) show a broad, but relatively homogeneous gaussian distribution for all types of signals and amplification systems. The variation of the intranuclear fluorescence intensity is very high up to $70 \%$ (Fig. 2c), whereas the area of the spots remains stable and less variable (Fig. 2d).

\subsection{Pepsin treatment}

In order to increase the signal-to-noise ratio, we investigated the effect of a pepsin treatment first on the signal characteristics obtained with the Samba ${ }^{\mathrm{TM}}$ system, then on the DISCOVERY ${ }^{\mathrm{TM}}$ scoring. 

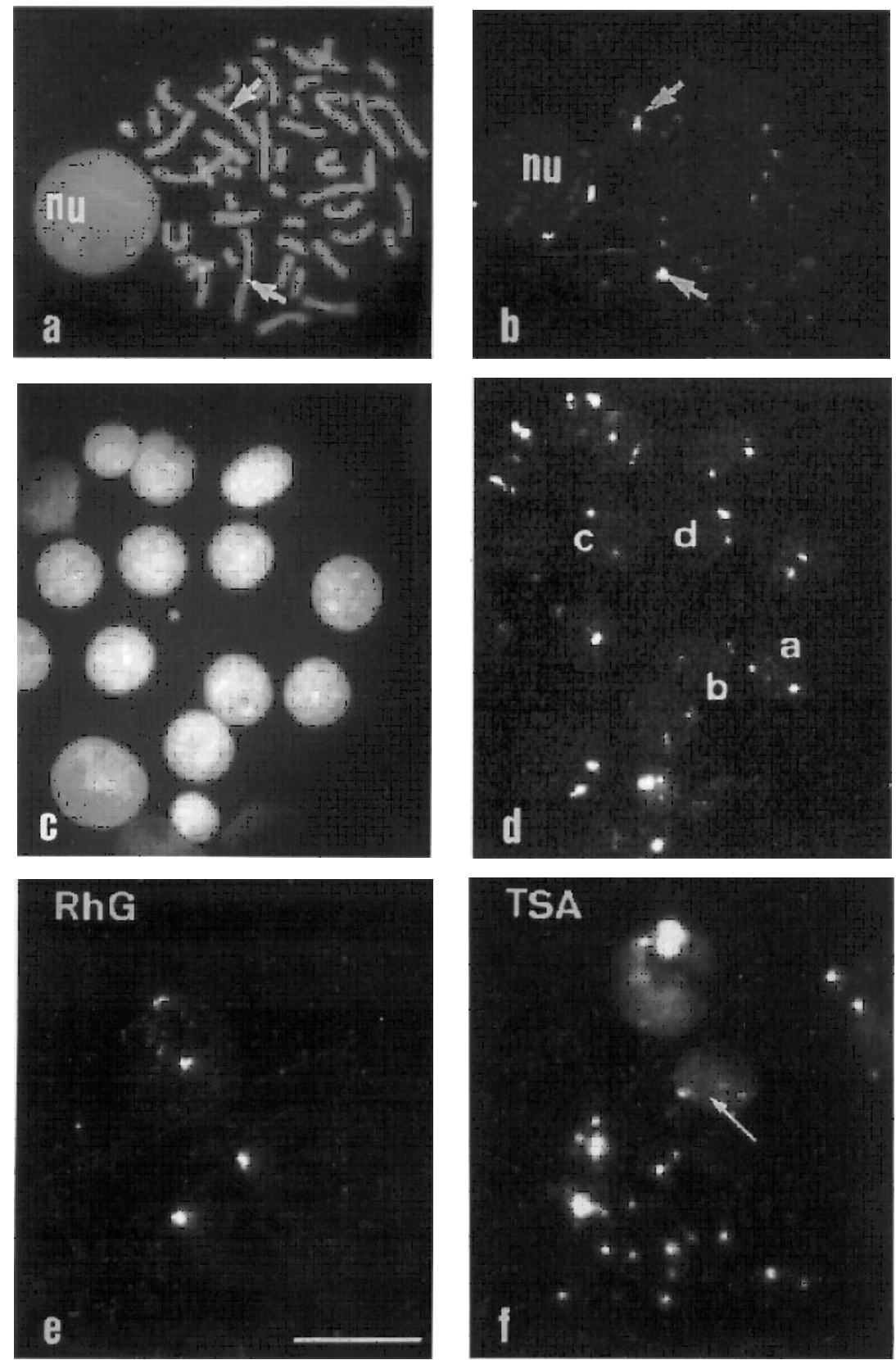

Fig. 1. FISH spots of the centromeric probe for chromosome 3 on metaphases and nuclei of stimulated lymphocytes. (a), (b) Hybridization specificity of the alphoid centromeric probe (arrows). (a) shows a metaphase and a nucleus after DAPI staining with two labeled chromosomes (arrows). (b) shows the principal signals detected with a FITC filter that are located over the two centromeric regions of the chromosome 3 and inside the nucleus. Minor signals are also detected using our high sensitive CCD camera whereas they are not visible when using the DISCOVERY ${ }^{\mathrm{TM}}$ system. (c), (d) Hybridization signals of the centromeric alphoid probe for chromosome 3 in interphase nuclei. (c) Field of DAPI-stained nuclei. Figure (d) same field using a FITC filter - many nuclei show two spots. The intensity of the spot fluorescence varies from one nucleus (a) to another (b) as well as inside one nucleus ((c) or (d)). (e), (f) Spot fluorescence using no amplification (e) or with TSA amplification system (f). After TSA, the spot area is very large and the nuclei show strong fluorescence. The heterogeneity of the spots within the same nucleus or between different nuclei is still observed (arrows). Scale bar $=50 \mu \mathrm{m}$. 
Table 1

Effect of pepsin treatment on manual scoring (30 nuclei)

\begin{tabular}{lccrrrrrrr}
\hline & 0 Spots & \multicolumn{3}{c}{ 1 Spot } & \multicolumn{2}{c}{ 2 Spots } & \multicolumn{2}{c}{$>2$ Spots } \\
\hline Pepsin treatment & - & + & - & + & - & + & - & + \\
FITC & 0 & 0 & 9 & 10 & 86 & 90 & 5 & 0 \\
Rhodol Green & 0 & 0 & 4 & 0 & 96 & 100 & 0 & 0 \\
$\begin{array}{l}\text { Amplified } \\
\text { Rhodol Green }\end{array}$ & 0 & 0 & 14 & 8 & 86 & 84 & 0 & 8 \\
TSA Rhodol & & & & & & & & \\
Green 1/250 & 0 & 0 & 18 & 14 & 82 & 86 & 0 & 0 \\
\hline
\end{tabular}

The shown numbers correspond to the percentages of the manual scoring.
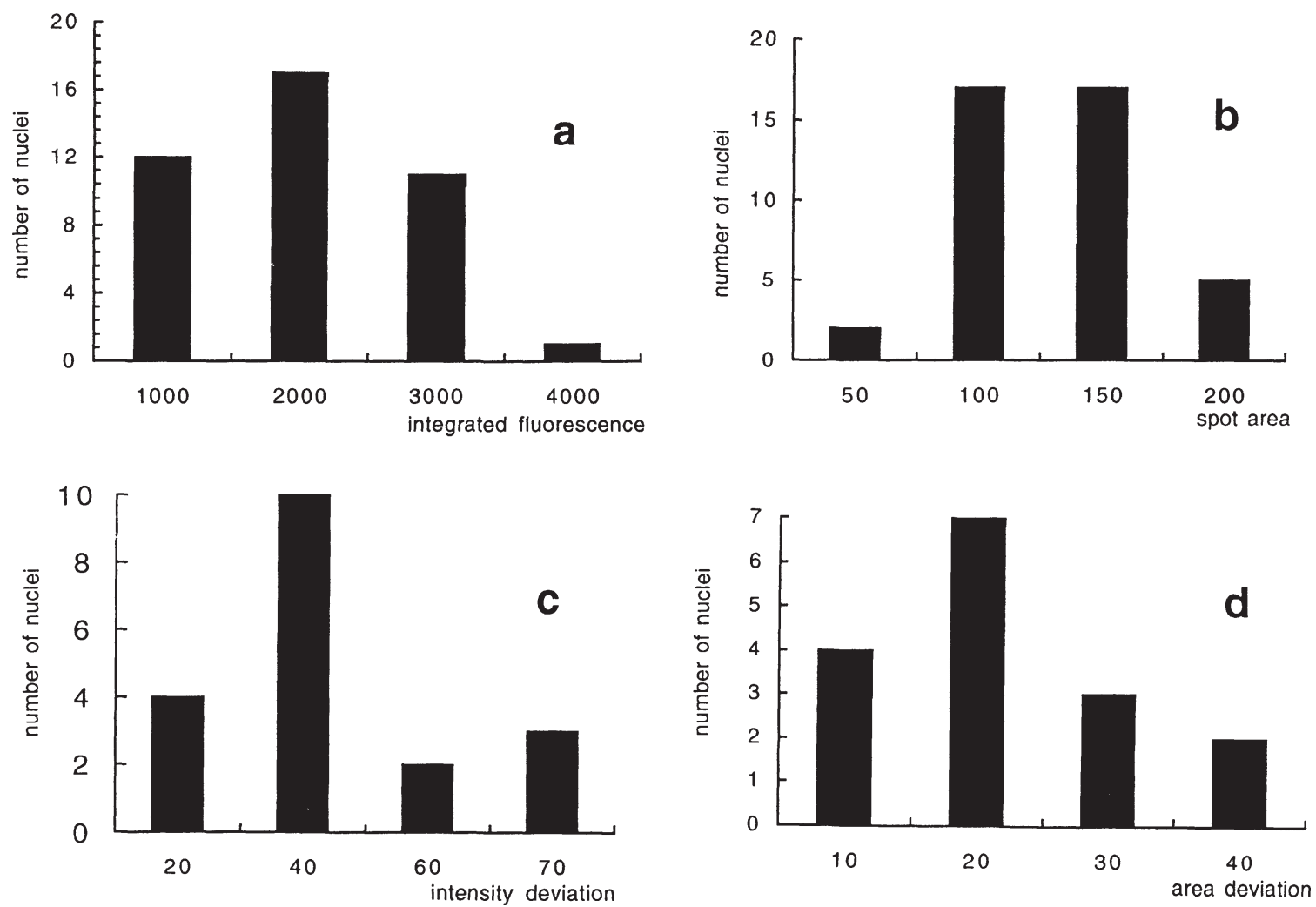

Fig. 2. Internuclear and intranuclear distribution of signal characteristics for 30 recorded nuclei (the shown example is for FITC, but is similar in all other cases). (a) Global (internuclear) distribution of the FISH spot intensity (in arbitrary units). (b) Global (internuclear) distribution of the FISH spot area (in pixels). (c) Intranuclear variation of spot intensities expressed in percentage of the standard deviation. (d) Intranuclear variation of spot areas expressed in percentage of the standard deviation.

The effect of the pepsin treatment on signal characteristics varied according to the detection mode (Table 2a). The intensity was most sharply increased for FITC while the spot area remained stable, and for TSA were both area and intensity decreased. The fluorescence profile of the intensity (Fig. 3) became sharper and higher in all cases. Background measurements performed on recorded images for TSA showed a twofold decrease (from 1506 to 880 arbitrary units) after pepsin treatment, whereas the background for the other systems was not significantly altered. 
Table 2a

Effect of pepsin treatment on signal characteristics

\begin{tabular}{lcccccccc}
\hline & \multicolumn{2}{c}{ Integrated fluorescence } & \multicolumn{2}{c}{ Mean intensity } & \multicolumn{2}{c}{$\begin{array}{c}\text { Maximum profile } \\
\text { intensity }\end{array}$} & \multicolumn{2}{c}{ Spot area } \\
\hline Pepsin treatment & - & + & - & + & - & + & - & + \\
FITC & $1630(795)$ & $4381(1790)$ & $15(5)$ & $40(10)$ & $28(15)$ & $75(30)$ & $112(44)$ & $111(29)$ \\
$\begin{array}{l}\text { Rhodol Green } \\
\text { Amplified }\end{array}$ & $1531(725)$ & $2780(1110)$ & $13(5)$ & $25(5)$ & $15(7)$ & $45(20)$ & $130(63)$ & $116(34)$ \\
$\begin{array}{l}R \text { hodol Green } \\
\text { TSA }\end{array}$ & $1693(1040)$ & $1619(1008)$ & $15(5)$ & $14(5)$ & $42(25)$ & $47(30)$ & $120(70)$ & $106(62)$ \\
$R$ hodol Green & $14737(3314)$ & $4148(2960)$ & $45(4)$ & $25(10)$ & $75(20)$ & $45(30)$ & $324(67)$ & $145(62)$ \\
\hline
\end{tabular}

Integrated fluorescence, mean intensity and maximum profile intensity are given in arbitrary units. Spot area is given in pixels. For TSA, the data corresponds to the minimum concentration of tyramide $(1 / 250)$. The values in brackets represent the corresponding standard deviations.

Table $2 b$

Effect of pepsin treatment on automated scoring

\begin{tabular}{lccccccccc}
\hline & \multicolumn{8}{c}{ Spot per nucleus } \\
\cline { 2 - 10 } & 0 Spots & \multicolumn{1}{c}{ 1 Spot } & \multicolumn{2}{c}{ 2 Spots } & \multicolumn{2}{c}{ Spots } \\
\hline Pepsin treatment & - & + & - & + & - & + & - & + \\
FITC & 20 & 19 & 32 & 31 & 41 & 40 & 7 & 10 \\
Rhodol Green & 10 & 11 & 33 & 25 & 44 & 40 & 13 & 24 \\
Amplified & & & & & & & & \\
Rhodol Green & 28 & 7 & 27 & 25 & 34 & 40 & 11 & 28 \\
TSA & & & & & & & & \\
$R$ hodol Green & 29 & 7 & 41 & 31 & 25 & 50 & 5 & 12 \\
\hline
\end{tabular}

The shown numbers correspond to the percentages of the automated scoring.

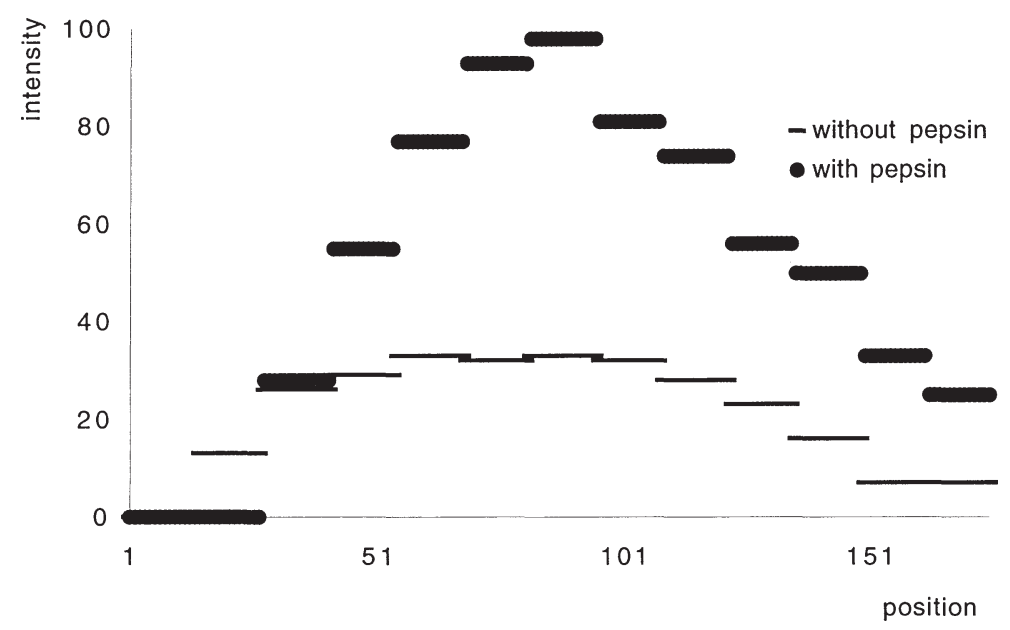

Fig. 3. Effect of pepsin on the fluorescence profile of the FISH spot, example of a FITC detected spot. The fluorescence intensity (in arbitrary units) is expressed as a function of the position on the spot diameter (in arbitrary units). 
Table 3

Effect of the tyramide concentration on signal characteristics

\begin{tabular}{lcccc}
\hline & \multicolumn{4}{c}{ Tyramide concentration } \\
\cline { 2 - 5 } & $1 / 250$ & $1 / 200$ & $1 / 150$ & $1 / 100$ \\
\hline Integrated fluorescence & $3157(2583)$ & $4612(2973)$ & $7329(4835)$ & $9837(7799)$ \\
Spot area & $100(40)$ & $135(60)$ & $155(84)$ & $200(115)$ \\
\hline
\end{tabular}

Concentrations are given dilutions of kit stock solution. The values in brackets represent the corresponding standard deviations.

Table 4

Effect of the tyramide concentration on automated scoring

\begin{tabular}{ccccc}
\hline TSA concentrations & 0 Spots & 1 Spot & 2 Spots & >2 Spots \\
\hline $1 / 100$ & 8 & 34 & 48 & 10 \\
$1 / 150$ & 4 & 28 & 53 & 15 \\
$1 / 200$ & 6 & 26 & 58 & 10 \\
$1 / 250$ & 14 & 29 & 47 & 10 \\
\hline
\end{tabular}

The shown numbers correspond to the percentages of the automated scoring.

The automated spot scoring using the DISCOVERY ${ }^{\mathrm{TM}}$ system (Table $2 \mathrm{~b}$ ) was affected in a different way. For the TSA slides, pepsin treatment improved the spot score. With pepsin treatment twice as many nuclei containing two spots were detected than without pepsin treatment (Table 2b). The percentage of nuclei containing 0 spots decreased significantly to $7 \%$ meaning probably a higher intensity of the signal. Using FITC and Rhodol Green detection, the spot count did not appear to be affected by pepsin.

\subsection{Comparison of the different amplification systems}

The first set of results concerns the comparison of FISH spots detected by Rhodol Green after amplification either by a standard streptavidin-biotin system or by the TSA system. Signal characteristics displayed in Table 2a (pepsin treated), show that TSA and FITC have the strongest integrated fluorescence. The amplified Rhodol Green was the weakest, even lower than standard Rhodol Green. The spot areas remained constant for all cases except for TSA where they were increased by $50 \%$.

Automated scoring (DISCOVERY ${ }^{\mathrm{TM}}$ ) for FITC, Rhodol Green and amplified Rhodol Green was similar (Table 2b, pepsin treated). The highest percentage of two-spot nuclei combined with the lowest number of no spots was achieved with the TSA system.

Signal characteristics of the TSA were observed to be directly correlated to the tyramide concentration (Table 3). The best results were obtained at concentrations between 1/150-1/200. At higher concentration, a decrease in the percentage of two-spot nuclei was observed (Tables 3 and 4).

\section{Discussion}

The results of the present study indicate the feasibility of automated FISH spot counting. The FISH standard protocol, however, needs to be improved for better scoring.

The influence of different fluochromes was first tested. The use of Rhodol Green gave the same results as FITC in terms of signal characteristics and automated scoring. Although Rhodol Green is 
more photostable than FITC, the integrated intensity was similar to that of FITC. In these experiments, $R$ hodol Green did not significantly improve the results contrary to what happens when recording with micrography. This lack of improvement may be due to the fact that both the CCD camera and the automated image cytometer use a relatively short illumination time of the sample compared to the observation time of a human observer working on a standard microscope. The photostability of the $R$ hodol Green may be more relevant in the latter case.

The effect of pepsin as a pretreatment prior to FISH affected the TSA system to the greatest degree, lowering the integrated fluorescence of the signals and improving the spot scoring drastically. Although these results seem to be contradictory, the most important effect of the pepsin was to halve the background noise, therefore increasing the sharpness of the signal and leading to a better score. This leads to better results due to an important reduction of background noise linked to the special deposition mode of the tyramide [1,2].

Biotin-streptavidin amplification was less effective than the standard procedure. The TSA system improved the automated scoring (percentage of two-spot nuclei), even at the lowest concentrations of tyramide. Spot detection improved up to a concentration of 1/200 after which the percentage of two-spot nuclei decreased and the percentage of one-spot nuclei rose. This is due to the fact that the DISCOVERY ${ }^{\mathrm{TM}}$ system does not seem to be able to separate very bright, overlapping spots at low magnification.

In summary, the TSA system combined with a pepsin pretreatment improved the automated scoring to give $58 \%$ of two-spot nuclei (650 counted nuclei). This value is relatively low compared to that obtained visually under the microscope or on recorded images $(80-100 \%)$. The choice of using brightfield microscopy instead of fluorescence microscopy $[3,11]$ already allowed better automated scoring (70\% of nuclei with two spots).

Although the latter results show a more realistic number of chromosome 3 present in interphase nuclei, they are far from representing the real hybridization efficiency. Continued development of techniques for the improvement of the automated detection is therefore necessary. It has been reported that including a borate or an acetic acid treatment $[7,11]$ increases the nuclear area and thereby improves expansion of the spots. Probes corresponding to more repeated sequences [7] may also be sometimes used, but are not always convenient.

Since signal characteristics show broad heterogeneity within a nucleus, signal improvement alone may not be sufficient. Technical development of the optical devices used by the automated image cytometer in combination with specimen preparation and staining is therefore necessary.

\section{Acknowledgements}

This work has been supported by grants from Becton-Dickinson (Leiden) and La Ligue Nationale contre le Cancer (Comité des Hauts-de-Seine).

\section{References}

[1] M.N. Bobrow, D.H. Thomas, K.J. Shaughnessy and G.J. Litt, Catalyzed reporter deposition, a novel method of signal amplification-application to immunoassays, Journal of Immunological Methods 125 (1989), 279-285.

[2] M.N. Bobrow, G.J. Litt, K.J. Shaughnessy, P.C. Mayer and J. Conlon, The use of catalyzed reporter deposition as a means of signal amplification in a variety of formats, Journal of Immunological Methods 150 (1992), 145-149. 
[3] L.J.A. Coignet, F.M. van de Rijke, M.F. Bertheas, A.K. Raap and H.J. Tanke, Automated counting of in situ hybridization dots in interphase cells of leukemia samples, Leukemia 10 (1996), 1065-1071.

[4] B. Dutrillaux and J. Couturier, Techniques d'obtention des préparations chromosomiques, in: La Pratique de l'Analyse Chomosomique, Masson, Paris-New York-Barcelona-Milan-Rio de Janeiro, 1981, pp. 9-18.

[5] R.E. Kibbelaar, F. Kok, E.J. Dreef, J.K. Kleiverda, C.J. Cornelisse, A.K. Raap and Ph.M. Kluin, Statistical methods in interphase cytogenetics: an experimental approach, Cytometry 14 (1993), 716-724.

[6] A.K. Raap, M.P.C. van de Corput, R.A.W. Vervenne, R.P.M. van Gijlswijk, H.J. Tanke and J. Wiegant, Ultra-sensitive FISH using peroxidase-mediated deposition of biotin- or fluochrome tyramides, Human Molecular Genetics 4(4) (1995), 529-534.

[7] P.S. Oud, C. van Egmond, E. Moerland, C.J. Cornelisse and N.W. Schipper, Automated fluorescent in situ hybridization spot counting with the DISCOVERY image analyzer, Cytometry (in press).

[8] D. Pinkel, J. Landegent, C. Collins, J. Fuscoe, R. Segraves, J. Lucas and J.W. Gray, Fluorescence in situ hybridization with human chromosome-specific libraries: detection of trisomy 21 and translocations of chromosome 4, Proc. Natl. Acad. Sci. USA 85 (1988), 9138-9142.

[9] D. Pinkel, T. Straume and J.W. Gray, Cytogenetic analysis using quantitative, high sensitivity, fluorescence hybridization, Proc. Natl. Acad. Sci. USA 83 (1986), 2934-2938.

[10] P.J. Poddinghe, F.C.S. Ramaekers and A.H.N. Hopman, Interphase cytogenetics of tumours, J. Pathol. 166 (1992), 215-224.

[11] F.M. van de Rijke, H. Vrolijk, W. Sloos, H.J. Tanke and A.K. Raap, Sample preparation and in situ hybridization techniques for automated molecular cytogenetic analysis of white blood cells, Cytometry 24 (1996), 151-157.

[12] R.P.M. van Gijlswijk, J. Wiegant, A.K. Raap and H.J. Tanke, Improved localization of fluorescent tyramides for fluorescence in situ hybridization using dextran sulfate and polyvinyl alcohol, Journal of Histochemistry and Cytochemistry 44(4) (1996), 389-392.

[13] J.E. Whitaker, R.P. Haugland, D. Ryan, P.C. Hewitt, R.P. Haugland and F.G. Prendergast, Fluorescent rhodol derivatives: versatile, photostable labels and tracers, Analytical Biochemistry 207 (1992), 267-279. 


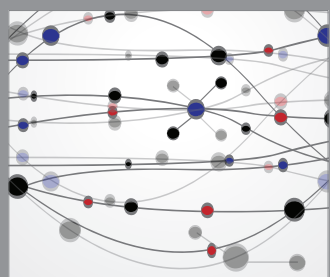

The Scientific World Journal
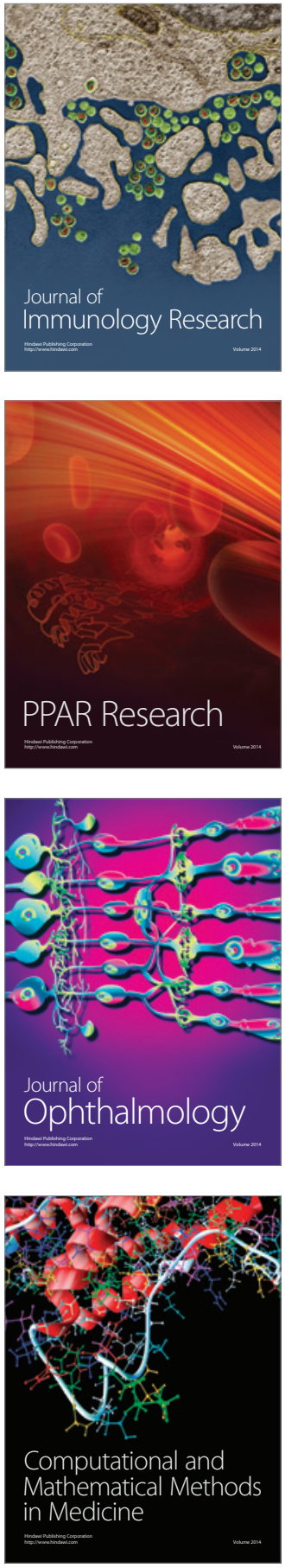

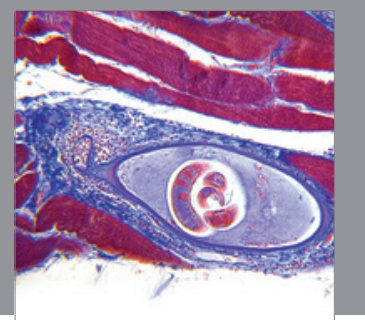

Gastroenterology

Research and Practice
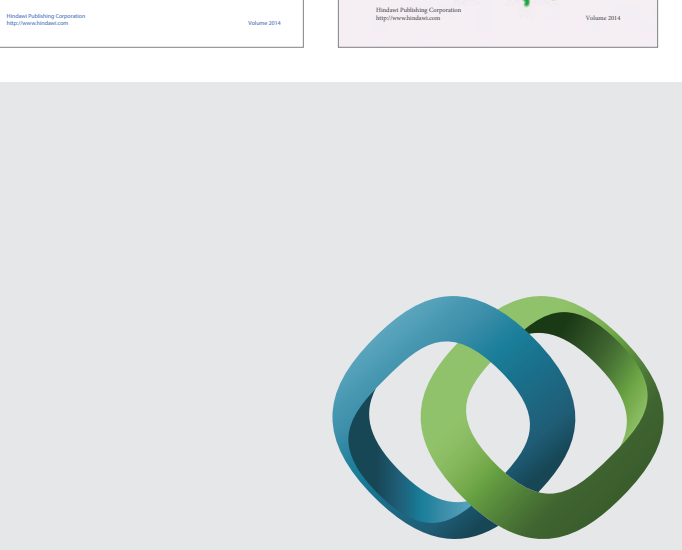

\section{Hindawi}

Submit your manuscripts at

http://www.hindawi.com
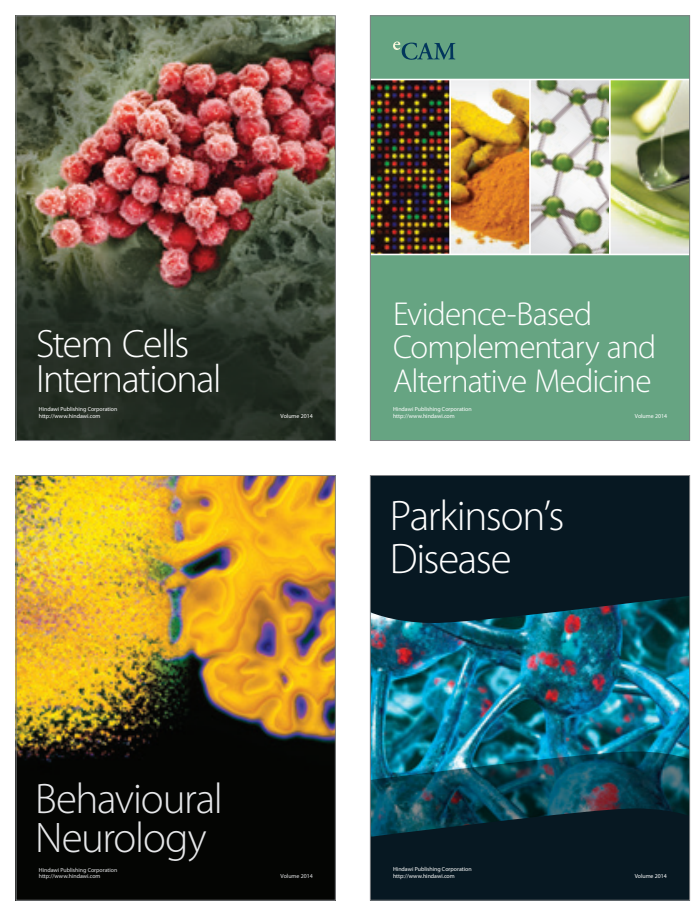

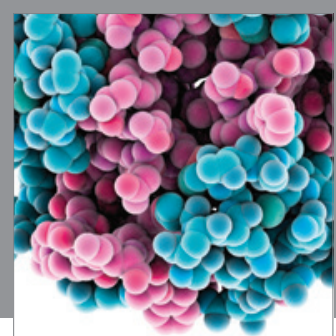

Journal of
Diabetes Research

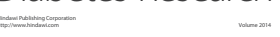

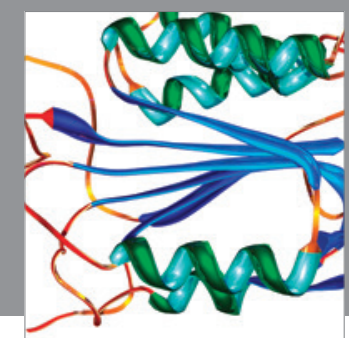

Disease Markers
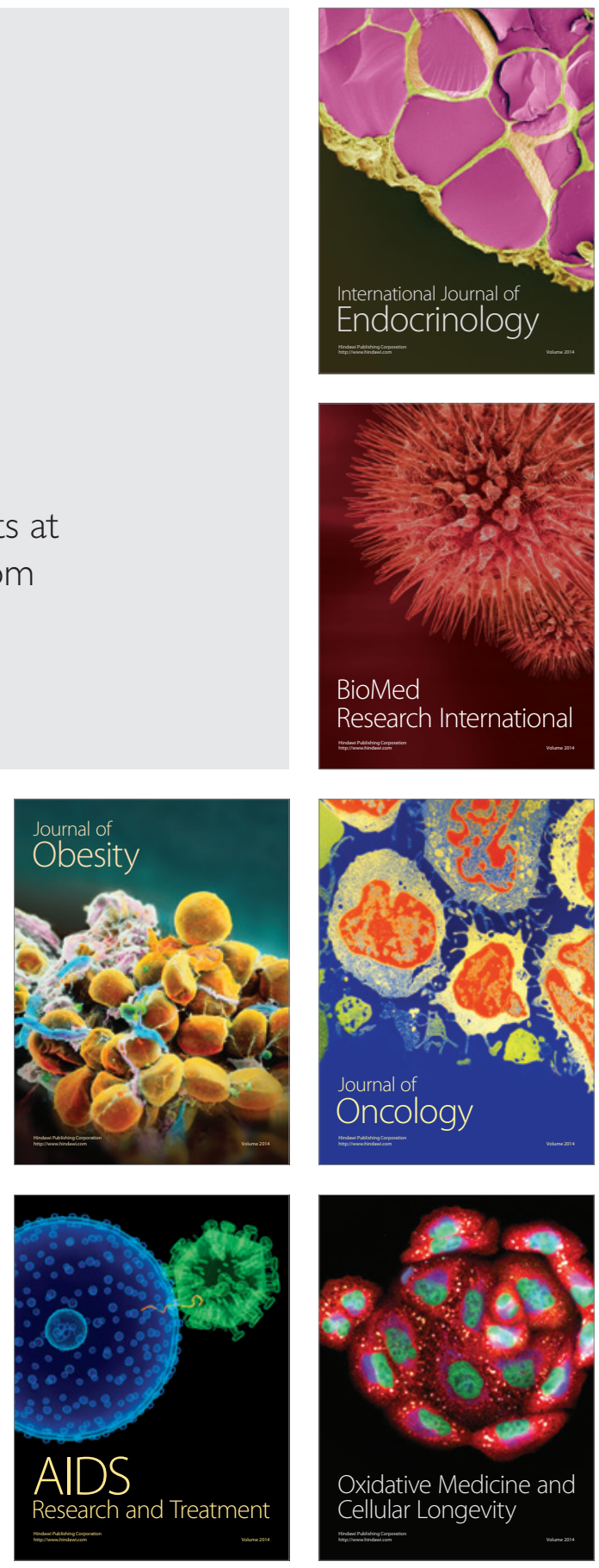